\title{
Approches linguistiques des langues spécialisées
}

\section{Pierre Lerat}

\section{OpenEdition}

\section{Journals}

Édition électronique

URL : http://journals.openedition.org/asp/2926

DOI : 10.4000/asp.2926

ISSN : 2108-6354

\section{Éditeur}

Groupe d'étude et de recherche en anglais de spécialité

\section{Édition imprimée}

Date de publication : 1 décembre 1997

Pagination : $1-10$

ISSN : 1246-8185

\section{Référence électronique}

Pierre Lerat, « Approches linguistiques des langues spécialisées », ASp [En ligne], 15-18 | 1997, mis en ligne le 16 avril 2012, consulté le 19 avril 2019. URL : http://journals.openedition.org/asp/2926 ; DOI : $10.4000 / a s p .2926$

Ce document a été généré automatiquement le 19 avril 2019.

Tous droits réservés 


\title{
Approches linguistiques des langues spécialisées
}

\author{
Pierre Lerat
}

1 Comme les langues spécialisées ne sont rien d'autre que des usages spécialisés des langues naturelles, leur étude suppose la prise en compte des différents niveaux de l'analyse linguistique.

2 Leur spécificité est essentiellement lexico-syntaxique, du côté des formes, et lexicoconceptuelle, du côté des contenus, d'où le besoin de théories appropriées. Les enjeux sont principalement l'aide à la traduction, à la rédaction, à la documentation et à l'apprentissage. Comme ces différentes activités seront de plus en plus assistées, il y a lieu de s'attacher en priorité à ce qui est gérable sous la forme de bases de données et mobilisable avec sécurité et rapidité.

3 Si l'on admet ces principes généraux, il faut en tirer quelques conséquences. La première est théorique : la meilleure conception d'ensemble est celle qui lexicalise au maximum la syntaxe et qui, en matière conceptuelle, tire le plus parti du jeu des relations typiques entre les mots. La seconde est méthodologique : la meilleure base de données est celle qui réunit les connaissances linguistiques et encyclopédiques dont ont besoin les usagers.

4 En privilégiant ainsi le lexique, on risque de réduire le langage à un jeu de contraintes formelles et logiques, au détriment de la dimension énonciative et pragmatique. L'objection serait forte si l'étude de cette dimension était plus avancée et avait obtenu des résultats indiscutablement scientifiques au sens ordinaire. Les sciences du langage, comme les autres, ne se constituent qu'en intégrant leurs résultats aux acquis provisoires des disciplines voisines et en s'en appropriant certains; or on est encore loin d'une situation où toutes les observations sur l'activité de langage, sur les textes et sur les situations d'énonciation constitueraient un corps de connaissances réellement systématisable.

5 Une autre limite du présent article résulte de son intitulé même, qui reflète un point de vue essentiellement descriptif : il est clair que l'exploitation didactique de cette mise en perspectives linguistiques appartient aux professionnels qui sont les professeurs du secteur LANSAD. Enseigner une LSP suppose des méthodes appropriées qui ne sont pas 
mon objet propre mais dont je ne méconnais pas l'importance. C'est pourquoi mon espoir est de susciter des idées d'intégration d'approches linguistiques à la méthodologie de l'enseignement des langues spécialisées.

\section{Fondements linguistiques}

6 Aucune théorie linguistique, quelle qu'elle soit, n'a jamais isolé le fonctionnement des langues spécialisées de celui des langues naturelles en général. Que l'on se tourne vers la théorie du signe issue de Saussure, vers la théorie de la proposition issue de la tradition logique, vers celle de la phrase au sens des grammaires formelles ou vers la théorie de l'énoncé élaborée depuis les années soixante, les avis convergent sur deux points essentiels : d'une part une langue spécialisée n'est pas une simple nomenclature, d'autre part la production de textes scientifiques et techniques suppose la mobilisation de compétences linguistiques plus larges. Il ne faut donc pas s'étonner des limites du rendement ni des méthodes audiovisuelles ni des expériences de type niveau-seuil.

7 Sur ce fond de compétence générale de langue et de communication, des tendances caractéristiques n'en sont pas moins remarquables. La principale est probablement l'importance particulière de l'écriture, y compris de l'écriture de signes non linguistiques, comme les formules propres à la notation dans telle ou telle science; je ne dirai rien ici de cette spécificité sémiotique, qui est étrangère à mon propos, mais elle est de grande conséquence. Les autres tendances sont observables aux différents niveaux de l'analyse de l'écrit, c'est-à-dire, dans la pratique, à chaque étape du travail sur les suites de chaînes de caractères.

\subsection{Niveau lexical}

8 Les unités lexicales spécialisées présentent par rapport aux autres les particularités suivantes.

9 - Elles utilisent la dérivation au bénéfice de la monosémie. Par exemple, homogénéiser et mélanger sont des verbes d'usage large : ce sont pour des humains ou pour des matériels des façons d'agir sur des humains ou des objets concrets ou abstraits, donc les sujets et les compléments possibles ne constituent pas des classes sémantiques; en revanche, en plomberie, un homogénéiseur est un type particulier de robinet, que l'on appelle aussi un mélangeur. Le sens, en pareil cas, n'est pas seulement dépendant de la connaissance de la langue, mais ici la langue ne fait que mettre de l'ordre dans la connaissance des choses. Il en va de même dans les sciences : hydrique est l'adjectif propre à parler de l'eau si et seulement si l'on a en vue l'eau en tant que corps chimique. Une conséquence pédagogique capitale résulte de cette propriété: l'enseignant de langue maternelle spécialisée gagne à être également spécialiste de la matière concernée. La situation est un peu différente en langue étrangère, mais le recours aux professionnels du domaine y est plus ou moins nécessaire aussi.

10 - Les expressions spécialisées utilisent à forte dose la composition nominale au bénéfice de la dénomination des objets techniques et scientifiques. Cette fonction de dénomination fait que le composé ne peut être validé comme dénomination que par un professionnel, même si sa reconnaissance et son analyse sont facilitées par des tests linguistiques et le repérage des candidats rendu possible par un simple calcul statistique portant sur les 
cooccurrences lexicales. Il faut bien voir en effet qu'un composé relevant du vocabulaire technique n'a pas de spécificité grammaticale par rapport à un composé non technique mais que sa structure dépend entièrement des propriétés typologiques générales de telle langue. Ainsi, notoirement, il est facile de reconnaître un composé dans une langue germanique, nettement plus difficile de le faire dans une langue romane; par exemple, formellement, rien n'indique que les expressions accident du travail et jour ouvrable sont lexicalisées en français, alors qu'il en va tout autrement pour Arbeitsunfall et Werktag en allemand.

11 - Dans les vocabulaires spécialisés, le lien entre le mot technique et sa racine tend à être distendu. C'est bien entendu le cas, tout particulièrement, de l'emprunt, mais aussi de la métaphore, et également du composé; ainsi, un arbre à cames est un arbre, mais seulement au sens technologique, et l'équivalent allemand Nockenwelle n'a qu'un rapport analogique avec l'idée de « vague » ou d'« onde » attachée à Welle.

\subsection{Niveau syntaxique}

12 Au niveau de la phrase complexe, les tendances sont à la fois bien connues et limitées à quelques particularités comme le style abstrait (obtenu notamment par des nominalisations et des adjectivations), le style impersonnel (qui dépend du jeu combiné des voix, des modes, des temps et des personnes) et le style explicite (où les articulations sont exprimées par des connecteurs logiques). Faute de travaux comparatifs systématiques, il est difficile de dire si ce sont vraiment les contenus qui jouent un rôle, ou si l'on n'est pas plutôt en face d'un mode d'énonciation particulier, celui de tout discours didactique, quelle que soit la matière exposée. Les professeurs ont avantage à ne pas trop dissocier le savoir et sa transmission, ni la transmission scolaire et l'apprentissage en situation; les linguistes, qui sont aussi bien souvent des professeurs, sont bien placés pour comprendre ces amalgames; il convient néanmoins qu'ils ne limitent pas l'énonciation technique à celle du cours ou du rapport de stage, et qu'ils aient en vue des pratiques plus vitales dans les entreprises, telles que le mode d'emploi, le devis ou le cahier des charges.

13 - À un niveau plus global, celui du texte, les instruments linguistiques d'analyse restent très limités : la " grammaire de texte » obtient peu de résultats en dehors du traitement des anaphores, la typologie des actes de langage manque encore de robustesse, la notion d'environnement cognitif est nécessaire mais peu technique pour le moment, et elle relève plus de la logique que de la syntaxe proprement dite.

14 - C'est au niveau de la phrase simple et du groupe de mots que les progrès de la linguistique formelle sont les plus remarquables à l'heure actuelle et les plus bénéfiques à l'étude des textes spécialisés. On l'a vu plus haut à propos des dénominations composées. On le voit mieux encore là où la sémantique se confond avec l'interprétation de suites syntaxiques, c'est-à-dire dans le jeu des prédicats et des arguments qui saturent les prédicats. Autrement dit, un intérêt particulier de la syntaxe des langues spécialisées est qu'elle a une grande pertinence dès que la construction du verbe, du nom ou de l'adjectif impose un type de complément spécifique ou, inversement, que les noms d'objets appellent des expressions prédicatives appropriées. Ce contrôle réciproque des mots dépendants et des mots régissants dans la phrase simple et dans le syntagme conduit à attacher une importance toute particulière à la distribution fine: celle des classes grammaticales, à un premier niveau, celle des traits de sous-catégorisation comme 
« humain » ou « concret ", à un deuxième niveau, celle des « classes d'objets » au sens de Gaston Gross, à un troisième niveau, celle enfin des classes réduites à une ou quelques unités lexicales compatibles (ainsi, sulfurique est difficilement employable autrement qu'à la suite immédiate d'acide). Par exemple, le patient contracte une hépatite, le client contracte avec l'assureur, le muscle se contracte : dans le premier cas l'objet appartient à une classe sémantique homogène, celle des maladies, dans le deuxième il s'agit nécessairement d'une personne juridique, dans le dernier le sujet est concret et matériel, sans propriété plus spécifique.

À titre d'exemple, la langue des assurances n'est pas seulement une nomenclature de dictionnaire spécialisé, mais un jeu de relations entre des acteurs, des opérations, des risques, etc. Sa maîtrise est celle des liens prévisibles entre d'une part des expressions prédicatives verbales, comme assurer, nominales, comme assureur, assurance et assuré, ou adjectivales, comme assuré, et d'autre part le cortège de leurs arguments typiques (assurer un bien, assurance sur la vie, véhicule assuré, etc.).

De façon plus élémentaire, parce que purement formelle, la distribution simplement grammaticale des mots, y compris celle des articles et des prépositions, est un guide indispensable pour la traduction. L'élaboration des systèmes de traduction automatique est éclairante pour l'aide à la traduction purement humaine, parce qu'ils exigent l'explicitation des contraintes de cooccurrence. Ainsi, il est sans utilité de dire que l'expression à terme est une locution, car cette information ne sert à rien pour la traduire en anglais ou en allemand; ce qui importe en pareil cas est de lui affecter une catégorie grammaticale précise car, selon qu'il s'agit d'un emploi adjectival ou adverbial la traduction, pour un même domaine de connaissances, celui des marchés financiers, sera différente. Une opération à terme sera a forward transaction, ein Termingeschäft, un cours à terme a forward rate, ein Terminkurs; acheter à terme sera to buy forward, avec une position postverbale, auf Termin kaufen, sans soudure et avec une préposition. Rien de tout cela ne va de soi, surtout pour un apprenant, un non-spécialiste ou un étranger même disposant déjà d'une certaine familiarité avec la langue.

\subsection{Niveau conceptuel}

17 Dans la tradition antimentaliste qui a prévalu autour du structuralisme, le linguiste n'était pas tenu d'avoir une théorie du concept, et seul le psycholinguiste était préoccupé de la compatibilité des travaux sur les langues et des travaux sur l'acquisition des connaissances. Le projet interdisciplinaire connu sous le nom de sciences cognitives a le mérite, entre autres, de rappeler la nécessité de privilégier les approches compatibles, d'une discipline à l'autre. À cet égard la terminologie, dès les années trente, a pris parti pour l'école viennoise de philosophie du langage, soucieuse des relations entre les mots et les choses à travers des conceptualisations, au risque de se couper pour un temps d'une linguistique devenue immanentiste avec Saussure. On peut maintenant reprocher à la philosophie analytique de ne pas avoir fait une part suffisante au sujet conceptualisant, et donc à la terminologie d'avoir privilégié un point de vue ontologique plutôt que culturaliste, mais au moins le terme est clairement la dénomination d'un concept depuis Wüster.

Comment définir le concept ? La définition la plus satisfaisante, selon les critères de la linguistique, ne peut pas être celle qui sépare le concept de sa dénomination dans une langue naturelle donnée, car ce serait méconnaître à la fois l'importance des différences 
de conceptualisations selon les cultures et celle de l'arbitraire relatif selon les racines lexicales utilisées. Il y a donc lieu, à mon avis, d'adopter une conception compatible à la fois avec les exigences de la linguistique et celle de disciplines connexes, à commencer par la psychologie. C'est ce que fait dans un ouvrage récent Sylvain Auroux (1996: 207). Pour cet auteur, le concept est un « réseau hétérogène d'informations dont le mot ne forme que l'un des composants, les autres étant les représentations sensorielles (visuelles, tactiles, olfactives) et les informations culturelles typiques (clé : instrument pour ouvrir/ fermer des portes) ».

On peut bien entendu formuler autrement la même idée. On retrouve ici, en particulier, les entrées lexicales et encyclopédiques de Sperber et Wilson (1986: 86), ce qui peut donner envie de prendre en compte aussi des entrées logiques, autorisant la déduction de concept à concept, ainsi que le font ces auteurs; au reste, l'exemple de "clé » signale, implicitement, une implication nécessaire, l'hyponymie de clé par rapport à instrument. Telle quelle, cette définition est adéquate pour rendre compte du statut particulier du terme : elle intègre au concept la dénomination (ou les dénominations), elle prend en compte des propriétés perceptuelles, comme la forme schématique des objets dans le Dictionnaire de la machine-outil de Wüster, et énumère les relations prédicatives typiques. Ainsi, une clé de serrure porte un nom, clé, elle a une forme dessinable, c'est un instrument qui a quelque chose à voir avec le concept de porte; de même, une clé à bougies a en commun avec la clé de serrure un élément de dénomination, clé, elle correspond à un objet de forme différente et à fonction également spécifique, puisqu'elle sert à visser et dévisser des bougies de véhicule automobile. Dans ces conditions, ce qui est important en langue spécialisée est le concept, entièrement différent selon les liens notionnels que permettent d'expliciter les relations avec les prédicats "ouvrir » et "visser » et avec les objets " porte» et «bougie », alors qu'en langue générale c'est le continuum qui est privilégié : visser, c'est une façon de serrer, le résultat est de fermer, et à ce compte les classes d'objets typiques ne sont plus que des cas particuliers d'un même schème. La différence est importante au point de vue méthodologique, car d'un côté l'essentiel est la logique de l'action sur le réel, dans l'autre c'est une logique du signifié immanent. Les langues spécialisées, à mon avis, imposent un point de vue encyclopédique, et donc une rupture avec la tradition du dictionnaire général unilingue, qui met l'accent sur les continuités en prenant le parti du mot et non pas celui du concept.

La sémantique du concept, à ce compte, est ainsi une sémantique entièrement relationnelle: relation entre dénomination et informations diverses, relations de généricité et de spécificité, relations prédicatives. La polysémie des expressions prédicatives correspond dans une large mesure à la variabilité des arguments qui vont avec, et celle des objets dépend des propriétés référentielles (appréhendables par la perception et la catégorisation). D'où la pertinence de la notion de domaine de connaissances: il y a clé et clé, selon qu'il s'agit de musique, de serrurerie ou de mécanique automobile. L'hyperonymie varie aussi avec les points de vue induits par les domaines de connaissances; le lait de vache est toujours du lait de vache, mais pour l'industriel c'est un produit, pour le médecin un aliment, pour le chimiste un liquide. Si la polysémie est facile à gérer dans une base de données prenant en compte des différences de domaines, la polyhiérarchie l'est moins; le problème se pose surtout pour la documentation, mais on le rencontre aussi dès qu'on essaie de concevoir une base de connaissances terminologiques multidomaine. 


\section{Une description d'emplois typiques}

\subsection{Pour une description limitée}

Paradoxalement, les langues spécialisées sont relativement simples, parce que les contraintes lexicales, sémantiques et pragmatiques y sont fortes. La prise en considération de domaines est quelquefois mal comprise, comme l'imposition artificielle d'oeillères alors que dans la communication réelle il y a du coq à l'âne, des niveaux de langue, des interactions variées etc. L'analyse d'interviews de techniciens ou celle d'articles de scientifiques à destination du grand public fournit des arguments aux tenants d'une "socioterminologie » qui ressemble beaucoup à une analyse de discours à entrée lexicale comme on en faisait dans les années 70 . Si l'on a en vue les connaissances capitalisées par une discipline à un moment donné et si l'on a le souci de représenter ces connaissances en vue de leur transmission, il faut bien accepter l'idée qu'il y a des disciplines et des sous-disciplines, des tâches imposant des procédures et une exigence de précision dans toute communication professionnelle. Là encore, la terminologie a été bien orientée dès ses débuts, en liant sa productivité à l'activité de normalisation ; conçue comme une linguistique d'ingénieur à l'origine, la terminologie a été pensée d'emblée comme instrument de résolution de problèmes. L'erreur a été de séparer les concepts de leurs expressions linguistiques au point de miser sur l'esperanto pour faciliter la communication et y accroître la sécurité. La vérité est que la communication professionnelle doit tenir compte des bizarreries des langues naturelles et tendre malgré tout vers la prévisibilité maximale, au risque d'utiliser une langue de travail unique, quand la sécurité des passagers d'un avion constitue un enjeu prioritaire.

\subsection{Pour une description fonctionnelle}

Un besoin de description systématique des langues spécialisées se fait sentir principalement en matière d'aide à l'apprentissage, à la rédaction, à la traduction et à la documentation.

Une vue trop étroite de l'apprentissage pourrait conduire à se passer d'informations phonétiques, graphiques, morphologiques et syntaxiques. Ce serait une erreur, car rien ne va de soi dans une matière aussi soumise à la prolifération des emprunts, des sigles et des néologismes de toutes sortes. De même, il faut prendre en compte des informations encyclopédiques et culturelles. Au total, mérite d'être pris en considération tout ce qui n'est pas prédictible à partir soit d'une compétence linguistique générale soit des connaissances de sens commun.

Pour la rédaction, il importe d'ajouter des connaissances portant sur les normes textuelles. Les instruments de référence ne sont pas seulement les correcteurs des traitements de textes, inspirés plus ou moins de manuels traditionnels, mais aussi, dans telle entreprise, telle feuille de style, tel formulaire (de contrat, par exemple) ou tel thesaurus permettant d'utiliser un vocabulaire contrôlé.

L'aide à la traduction requiert des ressources facilement accessibles. La traduction spécialisée supposant une bonne connaissance générale de deux langues, c'est sur le vocabulaire que les difficultés se concentrent. Elles exigent un traitement systématique des équivalences. Il y a toujours une solution mutatis mutandis, et ce qu'il faut expliciter ce 
sont les conditions de l'équivalence. C'est pourquoi je préfère la notion d'équivalence conditionnelle à celle de degré d'équivalence, le problème n'étant pas celui de la plus ou moins grande adéquation mais celui des conditions linguistiques et pragmatiques où il $\mathrm{y} a$ véritablement adéquation entre les formulations dans les deux langues.

Pour la documentation, l'important est la cohérence des thesaurus. Par rapport aux dictionnaires, les thesaurus se caractérisent par deux spécificités principales : d'une part le recours à un vocabulaire entièrement contrôlé pour dénommer les connaissances nécessaires dans un secteur d'activité, d'autre part la structuration de ces connaissances en termes de généricité/spécificité, de tout/partie, d'agents et d'objets typiques. Cette cohérence a un prix élevé : il est impossible d'en intégrer les informations dans un système de traduction automatique généraliste sans les avoir réévaluées pour cet usage externe. Par exemple, à Gaz de France prix du gaz désigne conventionnellement le prix d'achat que paie l'entreprise à ses fournisseurs, le prix de vente étant dénommé tarif du gaz. Pour le client, le tarif est bien sûr un prix, mais le thesaurus n'est pas fait pour les clients.

\subsection{Pour une description informatisable}

Il existe un instrument logiciel permettant de retrouver à la demande des informations si elles sont codées correctement : c'est le système de gestion de bases de données. Il est applicable à des objets linguistiques pour autant que ceux-ci sont clairement identifiés, d'où l'importance de la monosémie, qui est le lot des langues spécialisées, non des langues en général. Des mots pris dans un sens technique auront en commun, pour la langue prise en compte, un certain nombre d'attributs tels que la partie du discours, le genre grammatical, le nombre, les dérivés, les composés, le domaine, le cas échéant la classe d'objets, les prédicats appropriés, une définition, une note, une source, etc. Ces attributs prennent des valeurs en nombre limité (comme les genres et les nombres des substantifs ainsi que les domaines), ou au contraire extrêmement variables (comme les prédicats appropriés, les notes et les définitions). Seuls les cas de valeurs en nombre prédéterminé sont exploitables en matière de traitement automatique des langues, mais dans une base destinée à des usagers humains l'accès à une définition est très éclairant si la définition est bonne, et la mention de la source est une indication utile sur l'usage.

Les grands instruments disponibles, comme EURODICAUTOM et TERMIUM, ont été entrepris sans théorie linguistique. Inversement, les grands dictionnaires de langue informatisés, comme le Trésor de la langue française, sont pauvres en informations pertinentes sur le vocabulaire scientifique et technique. Enfin, les limites des systèmes de traduction automatique sont connues. Ils donnent satisfaction là où il s'agit de textes répétitifs, à syntaxe simple et à vocabulaire entièrement contrôlé. On obtiendra des résultats supérieurs en affinant les analyseurs syntaxiques et en mettant au point des analyseurs sémantiques au sens où l'entend Catherine Fuchs (in Piotrowski 1996 : 171). L'enjeu est d'arriver à " prédire, à l'aide d'un système de règles de filtrage mutuel, la valeur prise par chaque usage polysémique dans un énoncé ». On est très loin de pouvoir obtenir des succès spectaculaires dans le cas de mots courants tels que bon ou faire, mais les langues spécialisées se prêtent mieux à des filtrages mutuels entre mots conceptuellement solidaires dans un champ notionnel propre à un domaine. Par exemple, clôture est polysémique au sein même du droit, puisque susceptible d'une acception concrète (à propos de fossés ou de murs) ou d'une acception abstraite (à propos 
d'inscriptions ou de sessions), mais le prédicat approprié mitoyen permet un filtrage conditionnel; de même, portefeuille et action sont tous deux polysémiques, mais dans portefeuille d'actions les deux mots se désambiguïsent réciproquement.

\section{Conclusion} façon systématique de quelques propriétés conceptuelles ; ainsi, une assurance est un cas particulier de contrat, ou encore elle comporte des clauses. Ces relations ont une trace linguistique : l'hyperonymie de contrat se reconnaît à la vérité de propositions comme "une assurance est un contrat» et à la plausibilité d'anaphores comme «mon assurance... ce contrat »; de même, le syntagme clauses du contrat et la phrase le contrat comporte telle clause valident ensemble la relation partie-tout. Ainsi, la connaissance des choses se reflète en retour dans la langue.

Au niveau du texte, les assurances supposent l'usage réglé de formulaires et de lettrestypes. Il existe même un mot spécial, police d'assurance, pour dénommer un écrit contractuel propre à ce domaine. Ici la pragmatique prévaut, et la linguistique a peu de part dans les tests qui pourraient valider ces écrits : tests de complétude, en confrontant la police avec un modèle comportant exhaustivement les clauses nécessaires dans leur ordre procédural, tests de pertinence pour vérifier l'adéquation des données factuelles les unes avec les autres, de l'identité des contractants aux risques couverts. 


\section{BIBLIOGRAPHIE}

Auroux, S. 1996. La philosophie du langage. Paris : Presses Universitaires de France.

Gross, G. 1996. Les expressions figées. Paris : Ophrys.

Lerat, P. 1995. Les langues spécialisées. Paris : Presses Universitaires de France.

Piotrowski D. (dir.). 1996, Lexicographie et informatique. Paris : Didier Érudition.

Sperber, D. et D. Wilson. 1986. Relevance. Oxford : Blackwell.

Saussure, F. de. 1978 [1916]. Cours de linguistique générale, T. de Mauro (dir.). Paris : Payot.

\section{RÉSUMÉS}

Les langues spécialisées sont les langues naturelles face aux connaissances professionnelles. Les dénominations complexes, les distributions restreintes et les relations interconceptuelles sont trois caractéristiques majeures. La documentation et la traduction ont besoin de prendre en compte des domaines et des classes d'objets.

LSPs are natural languages used with reference to professional knowledge. Complex designations, restricted distributions and interconceptual links are the major features. Documentation and translation need to take into account domains and categories of objects.

\section{INDEX}

Mots-clés : classe d'objets, concept, dénomination, distribution, domaine, langue spécialisée

Keywords : category of objects, concept, designation, distribution, domain, specialised language

\section{AUTEUR}

\section{PIERRE LERAT}

Pierre Lerat est professeur de linguistique à l'Université Paris 13et a dirigé le Département des sciences de l'homme et de la société du CNRS. Il a consacré sa recherche aux questions de lexicologie, de lexicographie (générale et spécialisée, notamment juridique), de terminologie et de sémantique lexicale. Principal ouvrage: Les langues spécialisées, 1995 publié aux Presses universitaires de France. pierre.lerat@wanadoo.fr 